\title{
Characterization of OSSUT2 Expression and Regulation in Germinating Embryos of Rice Seeds
}

\author{
Wei Siao • Jia-Yi Chen • Hui-Hsin Hsiao • Ping Chung • \\ Shu-Jen Wang
}

Received: 15 July 2011 / Accepted: 9 September 2011 / Published online: 21 September 2011

(C) Springer Science+Business Media, LLC 2011

\begin{abstract}
OSSUT2 encodes a putative sucrose transporter containing 12 transmembrane domains in rice plants. Subcellular localization of the OsSUT2::GFP fusion protein indicated that OsSUT2 is a cell membrane protein. In embryos of germinating seeds, the expression of OSSUT2 gradually increased during the early germinating stage. The developmental regulations of OSSUT2 in germinating embryos could be mediated by sugars transported from endosperms. OSSUT2 expression was up-regulated by glucose through a hexokinase-independent pathway. Exogenous sucrose was sensed by a sensor localized on the plasma membrane and functioned as an enhancer to promote OSSUT2 expression. Based on OSSUT2 promoter::GUS expression in germinating seeds of transgenic rice, OSSUT2 was significantly expressed in the embryos and aleurone layers. In embryos, strong GUS expression was detected in the scutellum and vascular bundle tissues. Developmental stage- and sugar-dependent OSSUT2 expression was suggested to be controlled by transcriptional regulation of the promoter region.
\end{abstract}

Keywords Embryo Oryza sativa $\cdot$ Seed germination . Sucrose transporter

$\begin{array}{ll}\text { Abbreviations } \\ \text { DAI } & \text { Days after imbibition } \\ \text { Glc } & \text { Glucose } \\ \text { GUS } & \beta \text {-Glucuronidase } \\ \text { Man } & \text { Mannitol }\end{array}$

W. Siao $\cdot$ J.-Y. Chen $\cdot$ H.-H. Hsiao $\cdot$ P. Chung $\cdot$ S.-J. Wang $(\bowtie)$ Department of Agronomy, National Taiwan University,

No. 1, Section 4, Roosevelt Rd.,

Taipei 106, Taiwan

e-mail: shujen@ntu.edu.tw

$\begin{array}{ll}\text { MU } & \text { 4-Methylumbelliferone } \\ \text { 3-OMG } & \text { 3-O-Methylglucose } \\ \text { Pal } & \text { Palatinose } \\ \text { QRT-PCR } & \begin{array}{l}\text { Quantitative real-time reverse transcriptase } \\ \text { polymerase chain reaction }\end{array} \\ \text { Sc } & \text { Scutellum cells } \\ \text { Suc } & \text { Sucrose } \\ \text { SUT } & \text { Sucrose transporter } \\ \text { Ubi } & \text { Ubiquitin } \\ \text { V } & \text { Vascular bundle }\end{array}$

\section{Introduction}

In plants, carbohydrates translocate from the source to sink tissues through symplastic and apoplastic pathways. Sugar transport between cells in symplasts is mediated by plasmodesmata. In apoplasts, sugar translocators are responsible for carbohydrate uptake into cells. Because sucrose is the major transported form of carbohydrate between plant tissues, sucrose transporter (SUT) plays an important role in long-distance disaccharide transport. SUT1 in sugarcane functions to partition sucrose between the vascular bundle and storage cells (Rae et al. 2005). Maize SUT, ZmSUT1, is responsible for sugar uptake into phloem in source tissues and sugar unloading from phloem into cells in sink tissues (Carpaneto et al. 2005). Changes in carbohydrate allocation and the inhibition of photosynthesis have been observed in transgenic plants with reduced SUT activity due to antisense SUT genes; thus, SUT has been indicated to play an important role in carbohydrate partitioning and physiological processing (Kühn et al. 1996; Bürkle et al. 1998). 
To date, SUT genes have been identified from several plants, most belonging to gene families (Lemoine 2000; Lalonde et al. 2004). The SUT gene families of dicot and monocot species were classified into five sequence-based groups by Braun and Slewinski (2009). In rice, the SUT gene family consists of five genes, OSSUT1 to OSSUT5 (Aoki et al. 2003). OsSUT1 and 3 were classified into group 1. The group 1 SUT family is composed of several Poaceae SUT members; so far, no dicot SUTS have been found in group 1. OsSUT2 was classified with Arabidopsis SUT4 (AtSUT4) in group 2. AtSUT4 has been demonstrated to be responsible for the low-affinity/high-capacity transport system (reviewed by Lalonde et al. 2004). OSSUT4 and OSSUT5 were classified into group 3 and 5, respectively.

During plant development from seed germination to seed establishment and seedling growth, starch reserved in the seed is the primary carbon and energy source for sprouting and early seedling establishment, and then the sugar sources for later stages of seedling growth come from the photosynthetic shoots. In rice plants, carbohydrate transport from germinating seeds to other developing sink tissues is a fundamental process for plant development and growth. Sugar derived from starch degradation exported to the cytosol from storage organelles is the first step for longdistance carbohydrate translocation. In cereal endosperms, starch granules are attacked by $\alpha$-amylase (Murata et al. 1968), and the maltose and linear/short-branch-chain oligosaccharides are generated for further degradation by $\alpha$-glucosidase (Stanley et al. 2011). The hexoses produced by starch degradation can be taken up by the scutellum cells of the embryo. Hexoses have been suggested to be resynthesized into sucrose in the scutellum (Edelman et al. 1959; Nomura et al. 1969). Sucrose in the scutellum is loaded into the vascular bundle, transported, and then unloaded to growing tissues through apoplastic or symplastic pathways (Aoki et al. 2006). In addition, it was also suggested that sucrose transported from aleurone layers to endosperm at early germination stage could be further uptaked by scutellum (Aoki et al. 2006). Based on our previous work (Liu et al. 2010), soluble sugar can also be converted to starch in scutellum or the cells surrounding vascular bundles at the post-germination stage, and the amount of transitory starch in embryonic tissues was dependent on the demand of growing sink tissues. Phloem functions as an important pathway for carbon source transport among various plant tissues. In rice germinating seeds, OSSUT1 was the first OSSUT family member identified to have a tissue-specific expression pattern (Hirose et al. 1997; Scofield et al. 2007). Developmental expression and regulation of OSSUT1 has been observed in germinating seeds (Chen et al. 2010). However, the regulation of other members of the OSSUT gene family still needs to be established.
In the present study, we identified the subcellular localization of OSSUT2. The expression of OSSUT2 in germinating embryos was detected by real-time quantitative RT-PCR. The spatial and temporal transcriptional activities of the OSSUT2 promoter were detected in the germinating seeds of transgenic rice plants carrying the OSSUT2 promoter:: GUS fusion gene. Furthermore, the signal transduction of sugars for regulating OSSUT2 expression was examined and discussed.

\section{Materials and methods}

Plant materials and treatment

Rice (Oryza sativa L. cv. Tainung 67) seeds were obtained from the Hualien District Agricultural Research and Extension Station in Taiwan. For germination, seeds were sterilized in $2.5 \%$ sodium hypochlorite with Tween 20 for $20 \mathrm{~min}$ and subsequently washed with distilled $\mathrm{H}_{2} \mathrm{O}$ four times. Seeds were then germinated at $37^{\circ} \mathrm{C}$ in the dark for 3 days and then moved to the phytotron for growing at 30/ $25^{\circ} \mathrm{C}$ under natural daylight. To analyze the sugar content and OSSUT2 expression in embryos, the growing shoots and roots were cut and the embryos isolated after 1- to 5day seed imbibition.

To isolate embryos from dry seeds, the grain hulls were removed by machine and the embryos picked by razor blade. The isolated embryos were sterilized in $0.25 \%$ sodium hypochlorite for $10 \mathrm{~min}$ and subsequently washed with distilled $\mathrm{H}_{2} \mathrm{O}$ four times (Matsukura et al. 2000). For isolated embryo culturing, the embryos were placed on MS medium and incubated at $28^{\circ} \mathrm{C}$ in dark or light. For sugar and sugar analog treatments, the chemicals were added in MS medium. The concentration of all sugars and sugar analogs used in this study was $100 \mathrm{mM}$.

Homology analysis of amino acid sequences and transmembrane domain prediction

Multiple sequence alignments of SUT amino acid sequences were carried out using SDSC Biology WorkBench 3.2 (http://workbench.sdsc.edu/). The transmembrane domains on the OsSUT2 amino acid sequence were predicted according to the Hidden Markov Models using TMMOD software (Kahsay et al. 2005).

\section{RNA extraction}

Embryos isolated from ten seeds or harvested from medium were ground in liquid nitrogen, homogenized in $1 \mathrm{~mL}$ Trizol reagent (Invitrogen, Carlsbad, CA, USA), and centrifuged at $8,000 \times g$. The supernatant was treated with $0.2 \mathrm{~mL}$ chloro- 
form, shaken for $15 \mathrm{~s}$, and incubated at room temperature for $3 \mathrm{~min}$. After centrifugation at $12,000 \times \mathrm{g}$ for $15 \mathrm{~min}$ at $4^{\circ} \mathrm{C}$, the upper layer was transferred to a new tube. RNA was precipitated with $0.5 \mathrm{~mL}$ isopropanol and incubated for $10 \mathrm{~min}$ at room temperature. After centrifugation, the pellet was dissolved in $0.2 \mathrm{~mL} \mathrm{H}_{2} \mathrm{O}$. Before the gene expression analysis, the total RNA extracted from the embryos was treated with DNase to remove contaminating genomic DNA.

Quantitative real-time reverse transcriptase-PCR

Total RNA (200 ng) was used as the template for quantitative real-time RT-PCR analysis using the Brilliant SYBR Green QRT-PCR Master Mix (Stratagene, La Jolla, CA, USA), and PCR reactions were performed using a Multiplex 3000P RealTime PCR System (Stratagene). The gene-specific RT-PCR primers are listed on Table 1. RT-PCR was carried out as follows: $50^{\circ} \mathrm{C}$ for $30 \mathrm{~min}$ and $95^{\circ} \mathrm{C}$ for $10 \mathrm{~min}$, followed by 40 cycles of $95^{\circ} \mathrm{C}$ for $1 \mathrm{~min}, 58^{\circ} \mathrm{C}$ for $1 \mathrm{~min}$, and $72^{\circ} \mathrm{C}$ for $1 \mathrm{~min}$. To quantify relative gene expression levels accurately, the $C_{\mathrm{T}}$ values of $O S S U T 1$ to 5 were normalized to the $C_{\mathrm{T}}$ value of the ubiquitin (Ubi) gene. For all real-time RT-PCR analyses, three independent experiments were carried out, and the data are presented as mean $\pm \mathrm{SD}$.

Sugar analysis

Ten embryos were ground to powder in liquid nitrogen and extracted with $1 \mathrm{~mL}$ of $80 \%(v / v)$ ethanol at $80^{\circ} \mathrm{C}$ for $5 \mathrm{~min}$ before centrifugation at $3,000 \times g$. The supernatant was analyzed for glucose and sucrose following the method described by Spackman and Cobb (2002).

Subcellular location of OsSUT2

The coding region cDNA of OsSUT2 was cloned from rice embryos using RT-PCR. Total RNA was extracted from embryos 5 days after imbibition (DAI) and RT-PCR was performed using specific primers. The forward primer $\left(5^{\prime}-\right.$ T TCTAGAATGCCGCGGCGGCCTAGGCGG-3') contained the $X b a \mathrm{I}$ cloning site sequence, the reverse primer (5'-AGGATCCATCGGTGACCTCTCCTCCTTG$\left.3^{\prime}\right)$ contained the BamHI cloning site, and the stop codon sequence was not included. OSSUT2 cDNA was cloned inframe with the N-terminus of the green fluorescent protein (GFP) gene, driven by a CaMV35S promoter in a transient expression vector. The OSSUT2-GFP fusion construct was transiently expressed in barley aleurone layer cells mediated by a He Biolistic particle delivery system (model PDS-1000, Bio-Rad). Half-de-embryonated barley seeds were sterilized before soaking in a shooting buffer $(20 \mathrm{mM} \mathrm{Na}-$ succinate and $20 \mathrm{mM} \mathrm{CaCl}_{2}, \mathrm{pH} 5.0$ ) for $48 \mathrm{~h}$ at $24^{\circ} \mathrm{C}$ in the dark (Mena et al. 2002). Next, the pericarp was removed from the seeds and the aleurone layer exposed for bombardment. After bombardment, the seeds were incubated with shooting buffer for $24 \mathrm{~h}$ at $24^{\circ} \mathrm{C}$. Finally, fluorescence localization in the aleurone layer cells was observed using an AXIO Imager M1 fluorescence microscope (Carl Zeiss, Germany).

\section{Promoter cloning}

Rice genomic DNA was extracted from the leaf tissues with plant $\mathrm{DNA}_{\mathrm{ZOL}}$ reagent (Invitrogen). The OsSUT2 DNA fragment upstream of the translation start site, located from -830 to $-1 \mathrm{bp}$, was amplified by PCR using specific primers (forward primer with SacI site: 5'-GAGCTCT TAAGGAGCACCAA-3'; reverse primer with SmaI site: 5'-CCCGGGCTTCTTCTCGTGTT-3'). Putative cis-elements on the OsSUT2 promoter sequence were characterized by searching for similar motifs in the Database of Plant Cis-acting Regulatory DNA Elements (PLACE) (Higo et al. 1999). To generate the plasmid for the promoter activity assay in transgenic rice plants, the OsSUT2 promoter
Table 1 Primer pairs for realtime RT-PCR

\begin{tabular}{llll}
\hline Gene & Accession number & $\begin{array}{l}\text { Primer pair } \\
\text { (F: forward primer; R: reverse primer) }\end{array}$ & Amplicon size (bp) \\
\hline OsSUT1 & D87819 & F: 5'-CTGTGATTTTCCTGTCCCTG-3' & 136 \\
& & R: 5'-AACACTGCTAGTGGACCAGT-3' & \\
OsSUT2 & AB091672 & F: 5'-AGGAGGAGAGGTCACCGATAA-3' & 240 \\
& & R: 5'-CCAACATCCAATGTACAACAGCA-3' & \\
OsSUT3 & AB071809 & F: 5'-GCCCAAGGTCTCCGTCC-3' & 137 \\
& & R: 5'-TGCTATAGTACCCGCTCTAA-3' & \multirow{2}{*}{249} \\
OsSUT4 & AB091673 & F: 5'-TTTGGCTGAGCAGAACACCA-3' & \\
& & R: 5'-ATGTCATTCGGGCAGAGCTT-3' & \multirow{2}{*}{ F: 5'-CTAGTGCGAAACTCCATCAAA-3' } \\
& AB091674 & R: 5'-AAAATATTTGGGTTCCTGAGAT-3' & \\
Ubi & D12629 & F: 5'-CGCAAGTACAACCAGGACAA-3' & 101 \\
& & R: 5'-TGGTTGCTGTGACCACACTT-3' & \\
\hline
\end{tabular}


$(-830 /-1)$ was inserted into the SacI and SmaI sites of the pCHY10 vector (a gift from Dr. Chwan-Yang Hong), which contained the first intron of the Ubi gene fused to the $\beta$ glucuronidase $(G U S)$ reporter gene. We used restriction enzymes to isolate the DNA cassettes containing the OSSUT2 promoter fragment with the Ubi intron and GUS gene from the pCYH10 constructs. These cassettes were then ligated into the SacI and HindIII sites of the pCAMBIA1302 plasmid.

\section{Generation of transgenic rice plants}

To produce OSSUT2 promoter::GUS transgenic plants, the constructed pCAMBIA1302 plasmid was transformed into Agrobacterium tumefaciens EHA105. The cultured A. tumefaciens harboring the constructed plasmids were used to infect rice embryo-derived calli. The transformed calli were selected on medium containing $50 \mu \mathrm{g} / \mathrm{mL}$ hygromycin and $250 \mu \mathrm{g} / \mathrm{mL}$ cefotaxime. Finally, the transgenic rice plants were regenerated from the transformed calli (Hiei et al. 1994; Toki 1997).

\section{Histochemical GUS assay}

Histochemical GUS activity assays were performed as described previously (Jefferson 1987). Half-cut germinated seeds from transgenic plants were placed in a solution containing $10 \mathrm{mM}$ EDTA, $0.5 \mathrm{mM}$ potassium ferricyanide, $0.5 \mathrm{mM}$ potassium ferrocyanide, $1.0 \mathrm{mM}$ 5-bromo-4chloro-3-indolyl- $\beta$-D-glucuronide, $0.1 \%$ Triton $\mathrm{X}-100$, and $0.1 \mathrm{M}$ sodium phosphate buffer $(\mathrm{pH} 7.0)$ and incubated at $37^{\circ} \mathrm{C}$ for $4 \mathrm{~h}$. The staining reaction was stopped by adding $75 \%$ ethanol.

\section{Quantitative GUS activity assay}

Developmental regulation of the OSSUT2 promoter was analyzed in germinating embryos of transgenic rice seeds. Seeds were germinated in water containing hygromycin, and embryos were isolated from 3 and 5 DAI. To assay the effect of glucose on OSSUT2 promoter activity, the seeds were imbibed in hygromycin-containing water for 3 days and the germinated embryos were isolated from transgenic seeds. The isolated embryos were cultured in MS medium containing $100 \mathrm{mM}$ glucose solution for 5 days at $28^{\circ} \mathrm{C}$ in the dark. The protruding shoots and roots were excised and discarded before the embryo proteins were extracted with the buffer containing $100 \mathrm{mM}$ sodium phosphate $(\mathrm{pH} \mathrm{7.0)}$ ), $5 \mathrm{mM}$ dithiothreitol, $20 \mu \mathrm{g} / \mathrm{ml}$ leupeptin, and $20 \%(v / v)$ glycerol. Next, 4-methylumbelliferyl $\beta$-D-glucuronide was added as a substrate and GUS activities were measured fluorometrically in the embryos as described previously (Jefferson 1987).

\section{Results}

Subcellular localization of OsSUT2 protein

To characterize the protein structure and identify the subcellular localization of OsSUT2 protein, the OSSUT2 coding sequence was amplified by RT-PCR. The OSSUT2 cDNA (accession no. HQ875341) encodes a protein of 501 amino acids in length. According to membrane protein topology prediction using Hidden Markov Models in TMMOD software (Kahsay et al. 2005), the OsSUT2 protein contains 12 transmembrane domains (Fig. 1a, b). The amino- and carboxyl-terminal sequence tails predicted a cellular localization (Fig. 1b). Based on a previous phylogenetic analysis of the SUT gene family by Braun and Slewinski (2009), OSSUT2 was grouped with Arabidopsis AtSUT4. Furthermore, Kühn and Grof (2010) showed that AtSUT4 is also classified with StSUT4 and LeSUT4 in the same group. The amino acid identities between OsSUT2 and StSUT4, LeSUT4, and AtSUT4 are $66 \%, 66 \%$, and $64 \%$, respectively. The significant differences within the above SUT genes are at the amino terminus and central inside loop (Fig. 1a). In addition, the central inside loop of OsSUT2 (35 amino acids in length) is smaller than that of LeSUT2 (94 amino acid residues) and AtSUT2 (87 amino acid residues), which belong to the other SUT group (Fig. 1b). To determine the subcellular localization of OsSUT2 protein, the expression of OsSUT2GFP fusion protein was observed in the aleurone layer cells of barley seeds. Fluorescence imaging showed that the fusion protein was localized on the plasma membrane (Fig. 2).

Developmental expression of OsSUT2 in embryos during germination

To examine the expression of five OSSUT family genes in the embryos of germinating seeds, rice seeds were germinated in the dark for 3 days and then grown in phytotron with natural sunlight. The expression levels of OSSUT3 and 5 were lower than those of the other three OsSUT genes at 1 and 5 DAI (Fig. 3a). Quantitative RTPCR showed that the OSSUT2 transcript level was significantly higher than that of other OSSUT genes at 5 DAI (Fig. 3a). Among OSSUT1, 2, and 4, only the OSSUT2 mRNA of embryos was increased at 5 DAI compared to 1 DAI (Fig. 3a). Furthermore, the expression of OSSUT2 was observed to gradually increase during the early germination stage, and the transcript level at 5 DAI was 4.6-fold that of dry seed embryos (Fig. 3b). In addition, the expression of OSSUT2 in embryonic tissues increased with growth stage, even seedlings continuously grew in the dark after germination (Fig. 4a). However, the phenomenon of 
Fig. 1 Multiple amino acid sequence alignment and transmembrane domain analysis of SUT proteins. a Alignment of amino acid sequences from potato SUT4 (StSUT4; Genbank accession AF237780), tomato SUT4 (LeSUT4; AF176950), Arabidopsis SUT4 (AtSUT4; AY072092), and rice OsSUT2 (HQ875341). TM transmembrane domain. b Comparison of SUT protein structures. The inside and outside membrane regions and transmembrane domains of OsSUT2, StSUT4, AtSUT4, LeSUT4, AtSUT2 (AK226970), and LeSUT2 (AF166498) were predicted using TMMOD software.
A.

StSUT4 --MPEIERHRTRHNRPAIREP-- - - -VRP---RVPLRLLFRVASVAGGIQFGWA LQ LESUT4 --MPEIERHRTRHNRPAIREP-- - - - VRP---RVPLRLLLRVASVAGGIQFGWALQ ATSUT 4 MATSDQDRRHRVTRNRPPIARPSTSSSRPVVSPPRSRVSRRVLLRVASVACGIQFGWA LQ consensus ---mpeierhrtrhnrPai-ep-----vkp---rVp1R1L1RVASVA-GIQFGWA1Q St SUT4 LSLLTPYVQELGIPHAWASIIWLCGPLSGLLVQPLVGHMSDKCT-- -SRFGRRRPFIVAG LESUT4 LSLLTPYVQELGI PHAWASI IWLCGPLSGLLVQPLVGHMSDRCT---SRFGRRRPF IVAG OSSUT2 LSLLTPYVOELGI PHAFASLVWLC PLSGLLVOPLVGHLSDRIAPAA SPLGRRRPF IAAG consensus LSLLTPYVQELGI PHAWASI IWLCGPLSGL1VQPLVGHMSDkCt---SrfGRRRPFIVAG

AVSIMIAVLIIGF SADIGWLLGD--R-GEIKVRA IAAFVVGFWLLDVANNMTQGPCRALL ATSUT4 AVAISISVMVIGHAADIGWAFGD--REGKIRPRA IVAFVLGFWILDVANNMTOGPCRA OSSUT2 AASIAAAVLTVGSADLGRIFGDSITPGSTRLGAITAYLVGFWLLDVGNNATOGPCRAFP consensus A-sImiav1iigf sADiGW1-GD--r-GeikvrAIaAfVVGFW1LDVaNNmTQGPCRA11

StSUT4 ADLTQRDHRRTRVANAYFSLFMAIGNILGFATGSYSGWFKIFPFTLNTACTINCANLKAA LESUT 4 ADLTORDHRRTRVANAYF SLFMAIGNI LFATGSYS GWYRIFLFTLNTACTINCANLFA ATSUT4 ADLTENDNRRTRVANGYFSLFMAVGNVLGYATGSYNGWYKIFTFTRTVACNVECANLKSA ATSUT 1 ADLTENDNRRTRVANGYF SLFIAVGNVLGYATGSYNGWYKIFTFTRTVACNVECANLKSA

consensus ADLT--DhRRTRVANAYFSLFMAIGNILGEATGSYSGWYRIFPFT1-taCtinCANLR-A

StSUT4 FIIDIIFIATTTCISISAANEQPLDPSRGSSHTREEIGESSHGQEEAFLWELFGIFKYFP LESUT4 FILDIIFIATTTCISISAANEQPLDPSRGSSHTGEEIDESSHGQEEAFLWELFGIFKYFP ATSUT4 FYIDVVFIAITTILSVSAAHEVPLASLASEAHG---QTSGTDEAFLSEIFGTFRYFP

consensus FiidifiatTTcisisaanEqP1dpsrg--ht-eei-ess-gqeEAFLwE1FGiFkYFp StSUT4 GVVWVILLVTALTWIGWF PFLLFDTDWF GREIYGG---EPNDGRNYSAGVRMGS LGLMLN LESUT4 GVVWVILLVTA LTWIGWF PFLLFDTDWFGREIYGG-- - EPNDGKNYSAGVRMGS LGLMLN AtSUT4 GNVWIILLVTALTWIGWFPFILFDTDWMGREIYGG---EPNIGTSYSAGVSMGALGLLN OSSUT2 LPVWMVL IVTALTWIGWF PF ILFDTDWMGREIYRGS PDDPSITQSYHDGVRMGSFGLMLN consensus gVVWViL1VTALTWIGWFPF1LFDTDW-GREIYgG---ePn-gk-YsaGVrMGs1GLMLN SESUT4 SVLLGLTSLFMEKLCRKWGAGTWGVSNVVMSLCFIAMLIITAVRSNID-IGQGLPPDGI LESUT4 SVLLGLTSLFMEKLCRKWGA GFTWGVSNVVMSLCFIAMLIITAVRSNID-IGQGLPPDGI ATSUT4 SVFLGITSVLMEKLCRKWGA GFVWGISNI LMAICFLGMIITSFVASHLGYIGHEQPPASI consensus SVILGITS1fmEKLCRKWGAG-WGVSNVVM-1CFiaMliitav-snidyiggg1PPdgI

stsuT 4

LesUT4

$\begin{array}{ll}\text { ATSUT4 } & \text { VFAAVLIFTILGI PLAITYSVPYALISIRIESLGLGQGLSLGVLNLAIVIPQVIVSVGSG } \\ \text { OSSUT2 } & \text { VIASLVVFTILGAPLAITYSIPYAMAASRVENLGLGQGLAMGILNLAIVIPQVIVSLGSG }\end{array}$

$\begin{array}{ll}\text { ATSUT4 } & \text { VFAAVLIFTILGIPLAITYSVPYALISIRIESLGLGQGLSLGVLNLAIVIPQVIVSVGSG } \\ \text { OSSUT2 } & \text { VIASLVVFTILGAPLAITYSIPYAMAASRVELGLGQGLAMGILNLAIVIPQVIVSLGSG }\end{array}$

consensus VIAa1VVFSILGI PLAITYSVPYAIVSSRIEALGLGQGLSmGVLNLAIV-PQIVVS1GSG

STSUT4 PWDELFGGGNSPAFVVALSAFAGGLIAILAIPRTRVERPKIFA-

LESUT4 PWDELFGGNSPAFVVAALSAFAAGIAILAIPRTRVERPKIFA-

ATSUT4 PWDQLFGGGNSPALAVGATGFIGGIVAILALPRTRIQRPIPLP-

OSSUT2 PWDQLFGGNAPAFAVAAAASFIGGLVILGLPRARIASRRRGHR

consensus PWD-LFGGGNSPAf-VaA-saF-gGliAILaiPRtRvekpkifa-

B.
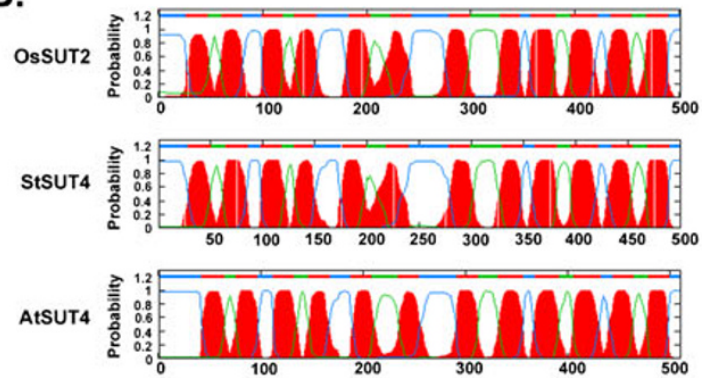

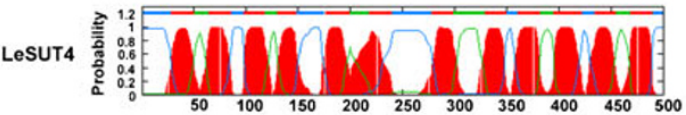

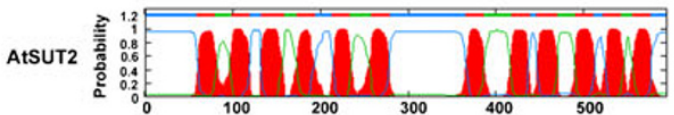

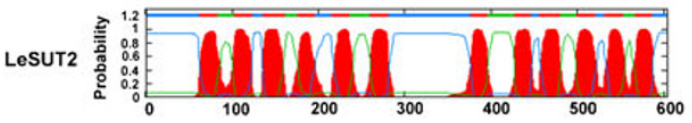

Amino acid No.

Transmembrane Inside Outside 
Fig. 2 Subcellular localization of OsSUT2 by transient expression of OsSUT2-GFP fusion proteins in barley aleurone layer cells. a Bright field image. b GFP fluorescence image. c Merged field and fluorescence image.
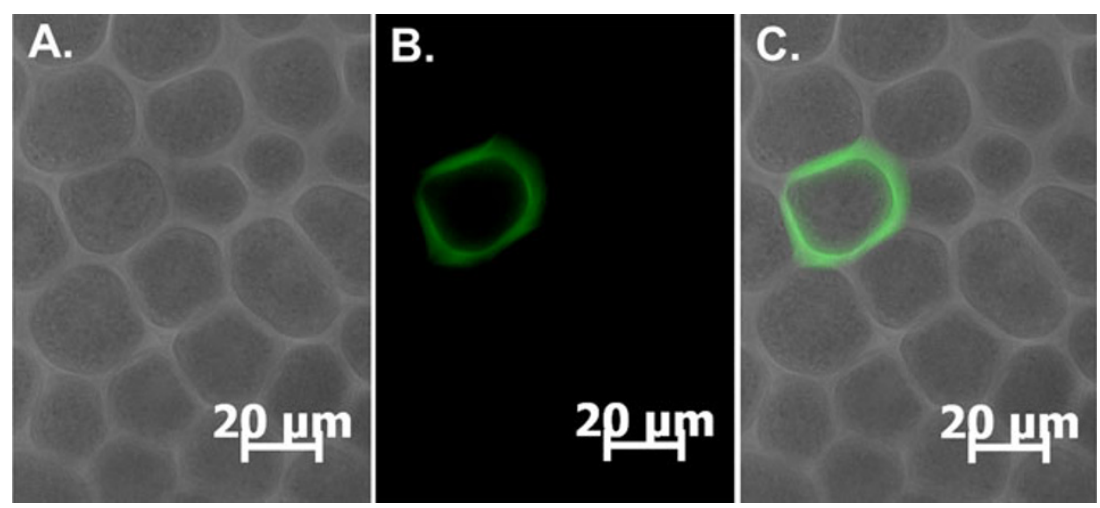

OSSUT2 up-regulation during germination was not obvious in dark-cultured isolated embryos (endosperm-free) (Fig. 4c).

Expression of OSSUT2 regulated by exogenous sugars

OsSUT2 expression in germinating embryos was different from those with and without endosperm status. It was

A.

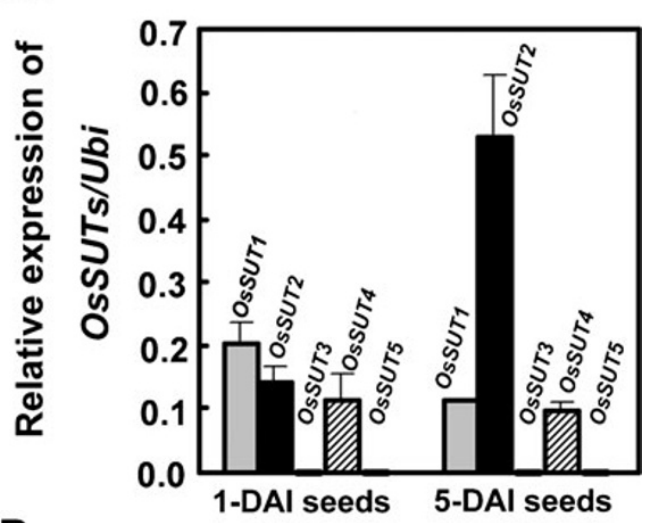

B.

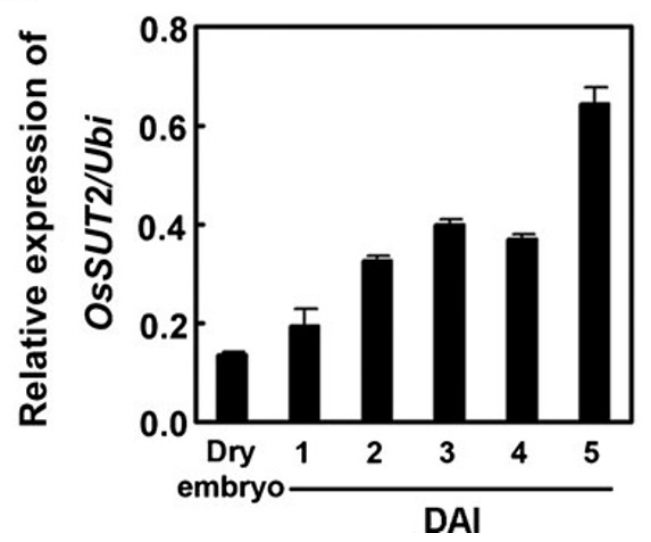

Fig. 3 Developmental expression of rice OSSUT in the embryos of germinating seeds. a Comparison of the expression of five OsSUT genes in the embryos of 1-DAI and 5-DAI seeds. b Changes in OSSUT2 expression in embryos during seed germination. The data are presented as mean $\pm \mathrm{SD}$. considered whether the sugar transported from endosperm during seed germination plays a role in the regulation of OsSUT2 expression in embryos. To evaluate this possibility, the levels of glucose and sucrose were analyzed in the embryos of germinating seeds from 0 to 5 DAI. Glucose levels gradually increased with the days after imbibition, but sucrose content fluctuated (Fig. 5). The changes in glucose content corresponded to the expression pattern of OsSUT2 transcripts in embryos during seed germination (Figs. 3b and 5). Even for seed germination in the dark, changes in glucose content and OSSUT2 transcript levels were consistent (Fig. 4a, b). In order to determine whether sugars are the factors up-regulating $O S S U T 2$ expression in the embryos of germinating seeds, the embryos isolated from dry seeds were cultured on sugar-containing MS medium in the dark for 5 days. Although glucose $(100 \mathrm{mM})$ and sucrose $(100 \mathrm{mM})$ slightly enhanced OSSUT2 mRNA levels in 1-day sugar-treated embryo samples, the effect was not significant (Fig. 6a). In 5-day cultured embryos, the OSSUT2 expression was obviously up-regulated by both glucose and sucrose, but not by the same concentration of mannitol (Fig. 6b).

Sugar analogs were applied to study the sugar-sensing pathway for regulating OSSUT2 expression. 3-O-Methylglucose (3-OMG), a nonmetabolizable glucose analog, was taken up by cells but was not phosphorylated by hexokinase (Dixon and Webb 1979). The results showed that 3-OMG (100 $\mathrm{mM})$ had the same effect as glucose $(100 \mathrm{mM})$ to enhance OSSUT2 expression (Fig. 7a). Palatinose, a nonmetabolizable sucrose analog, cannot be imported into plant cells (Bouteau et al. 1999). The effect of palatinose (100 mM) on OsSUT2 gene expression was similar to that of sucrose (100 mM) (Fig. 7b), suggesting that embryo cells sense sucrose signals to regulate OSSUT2 expression through a membrane sensor.

OsSUT2 promoter::GUS expression in embryos

Transgenic rice plants harboring the OSSUT2 promoter:: GUS construct was used to investigate the spatial expres- 
A.

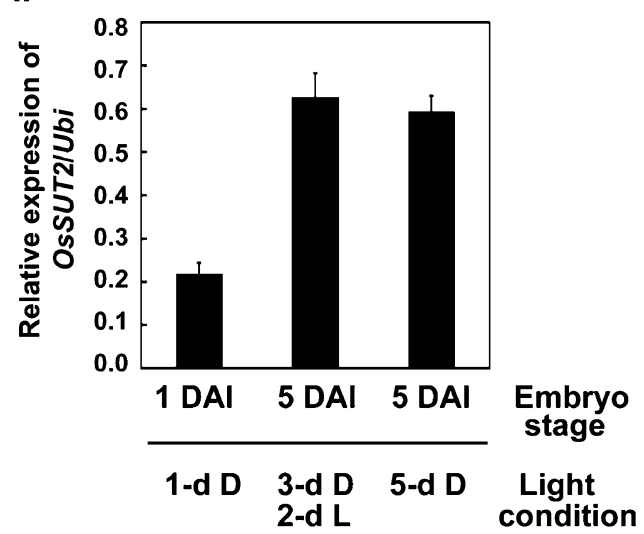

B.

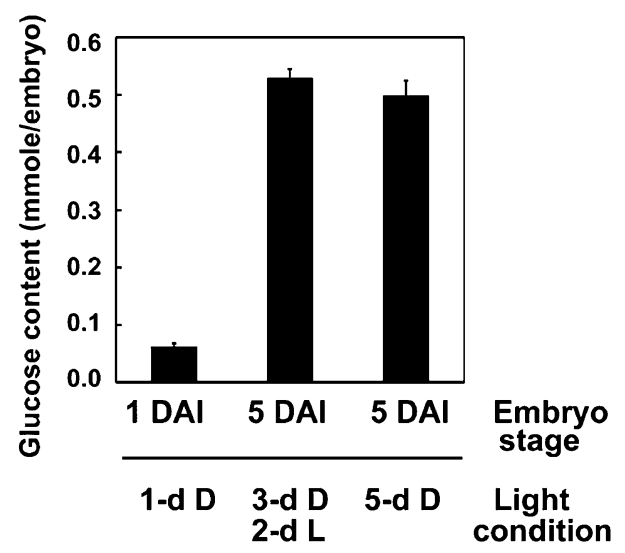

C.

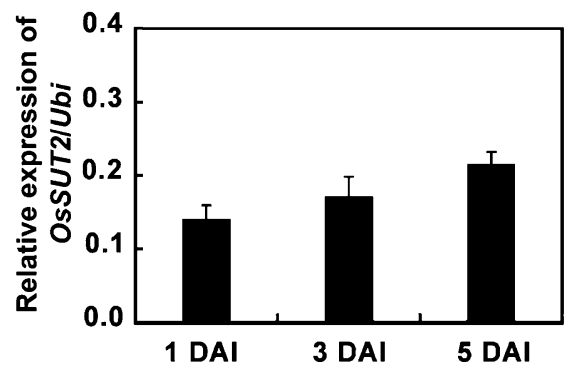

Fig. 4 OSSUT2 expression and glucose content in embryonic cells from light- and dark-grown seedlings. Rice seeds were germinated in the dark for 3 days. Some germinated seeds were moved to a phytotron with natural daylight for 2 days (labeled 3-day D/2-day L), and some germinated seeds were kept in the dark (labeled 5-day D) for growth. The seedlings were collected at 1 and 5 DAI, and the embryos were isolated for OSSUT2 expression analysis (a) and to measure glucose content (b). c The OSSUT2 expression in germinating isolated embryos was analyzed. The embryos were dissected from dry seeds and dark-grown in MS medium. OSSUT2 expression was detected after 1,3 , and 5 days of culture. The data are presented as mean $\pm \mathrm{SD}$

sion of OSSUT2 in rice embryos. Rice seeds of three independent transgenic lines were germinated in water, and GUS expression in germinating seeds was observed. GUS activities were performed in aleurone layers and embryos. In embryos, significant GUS staining was detected in the

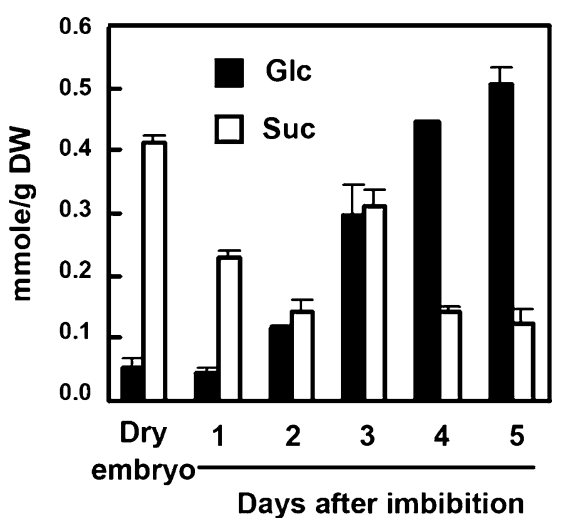

Fig. 5 Changes in the glucose and sucrose content of embryos during seed germination. The data are presented as mean $\pm \mathrm{SD}$. Glc glucose, Suc sucrose.

vascular tissues and scutellum cells of embryos (Fig. 8). In addition, the quantitative GUS activity assay showed that the GUS activity in embryos from 5-DAI seeds was significantly higher than that of 3-DAI seeds (Fig. 9a). To identify the effect of glucose on OSSUT2 promoter activity, the germinated embryos of transgenic rice seeds were isolated and incubated in $100 \mathrm{mM}$ glucose solution for
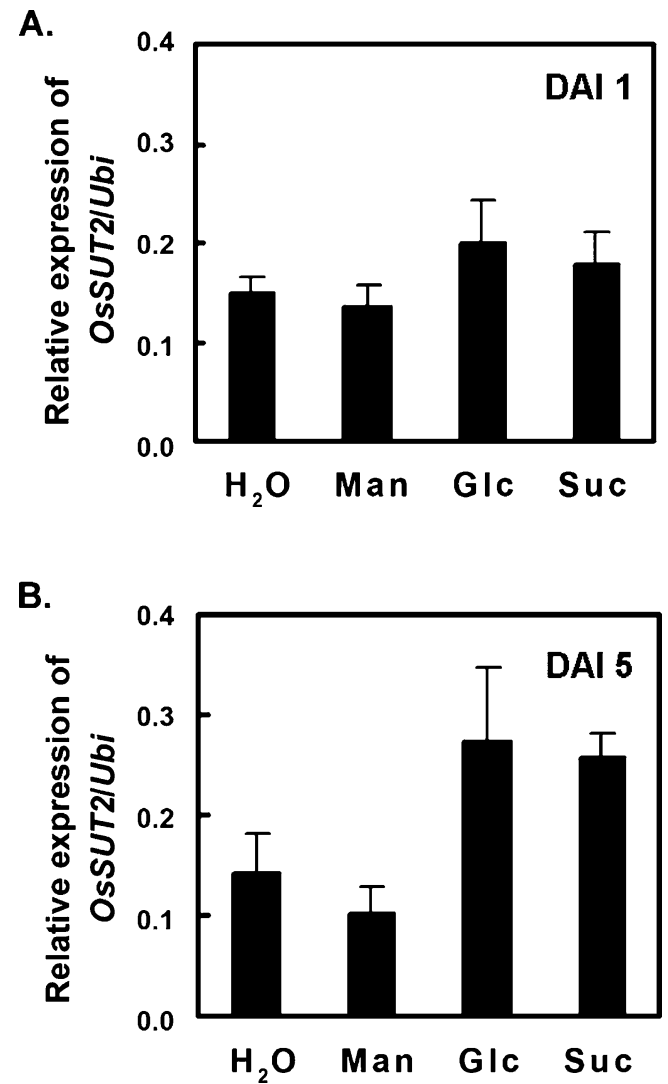

Fig. 6 Effects of sugars on OSSUT2 expression. OsSUT2 transcript levels were determined in isolated embryos cultured in medium containing mannitol (Man), glucose $(G l c)$, or sucrose (Suc) for 1 day (a) and 5 days (b). The data are presented as mean $\pm \mathrm{SD}$. 
A.

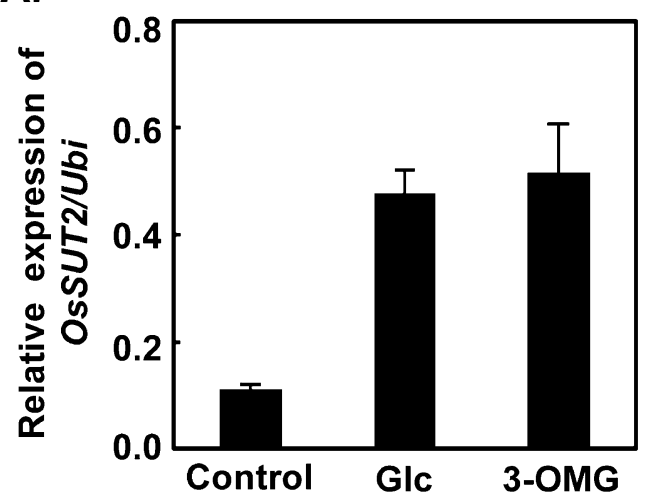

B.

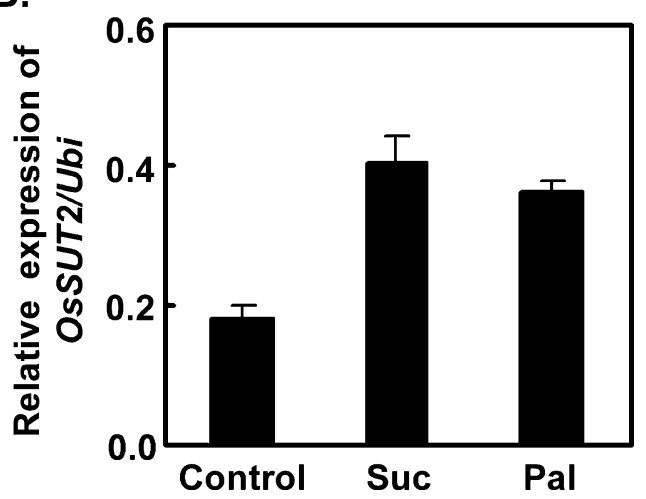

Fig. 7 Sugar-sensing pathways for OsSUT2 gene regulation in germinating embryos. a Effect of glucose analog 3-O-methylglucose $(3-O M G)$ on $O S S U T 2$ expression after 5 days of treatment. b Effect of the sucrose analog palatinose $(\mathrm{Pal})$ on $\mathrm{OSSUT} 2$ expression after 5 days of treatment. The data are presented as mean $\pm \mathrm{SD}$.

5 days. The OSSUT2 promoter activities were obviously enhanced (Fig. 9b).

\section{Discussion}

SUT proteins are important carriers for transporting sucrose across the plasma membrane or vacuolar membranes (reviewed by Kühn and Grof 2010). Arabidopsis SUT protein has also been found on the chloroplast membrane (Rolland et al. 2003). Rice OsSUT2 is classified in the same group with Arabidopsis AtSUT4, tomato LeSUT4, potato StSUT4, Lotus japonicus LjSUT4, and barley HvSUT2 (Braun and Slewinski 2009; Kühn and Grof 2010). Some of the above-mentioned SUTs, including AtSUT4, StSUT4, and LjSUT4, have been identified as low-affinity/high-capacity transporters (Weise et al. 2000; Reinders et al. 2008). In addition, AtSUT4, LjSUT4, and HvSUT2 are vacuolar transporters according to a previous analysis of transient SUT-GFP fusion protein expression (Endler et al. 2006; Reinders et al. 2008). On the other hand, LeSUT4 and StSUT4 are located on the plasma
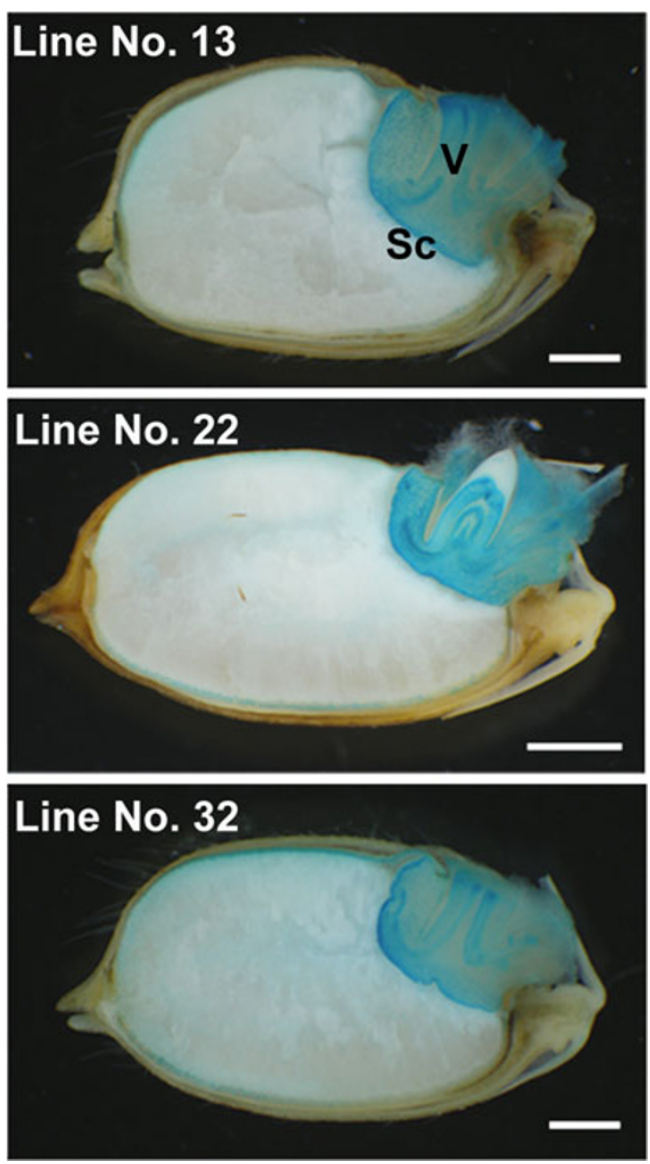

Fig. 8 Histochemical localization of $\beta$-glucuronidase activity in the embryos of transgenic rice plants harboring the OSSUT2 promoter:: GUS constructs. The seeds of transgenic rice lines 13, 22, and 32 were germinated in the dark for 3 days, and then the GUS activity was analyzed in half-cut germinated seeds. $S c$ scutellum cells, $V$ vascular bundle. Bar=1 $\mathrm{mm}$.

membrane of sieve elements (Reinders et al. 2002). OsSUT2 contains 12 transmembrane domains and was localized on plasma membrane. Amino acid alignment showed that the number of amino acids in the central inside loops are similar among OsSUT2 and other SUTs in group 4 (according to the classification by Braun and Slewinski 2009) (Fig. 1); however, the length of the OsSUT2 central loop is shorter than that of SUTs belonging to group 2, i.e., AtSUT2 and LeSUT2. LeSUT2 is considered to function as a putative sucrose sensor (Barker et al. 2000). The conserved domains in the extended cytoplasmic loop of LeSUT2 and AtSUT2 play an important role in signal sensing and transduction (Barker et al. 2000). Because the lengths and amino acid sequences of the central loops are significantly different between OsSUT2 and LeSUT2, the regulatory mechanism and function of OsSUT2 might not be completely identical to that of LeSUT2.

Carbohydrate transport from endosperms and embryos to coleoptiles, shoots, and roots is an important process for supplying developing tissues with a carbon source during 

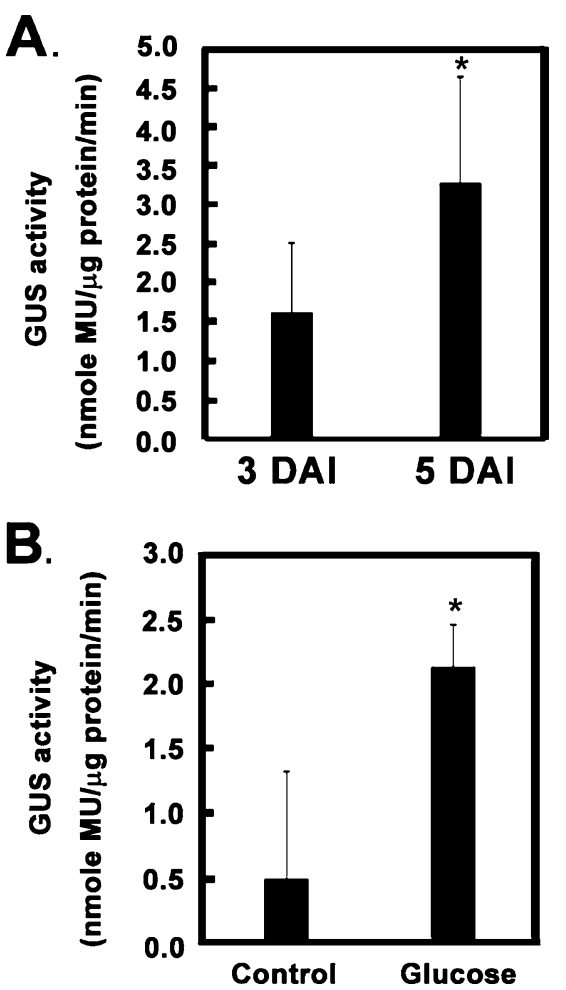

Fig. 9 Developmental regulation and effect of glucose on OSSUT2 promoter activity. a GUS activity was quantitatively analyzed in the embryos from germinating seeds of transgenic rice carrying the OSSUT2 promoter::GUS construct. b GUS activity was determined in isolated embryos cultured in medium with or without glucose. $M U$ 4methylumbelliferone. The data are presented as mean $\pm \mathrm{SD}$, and statistical significance is set at $p<0.05$ (asterisk).

seed germination and seedling establishment. The levels of OSSUT2 mRNA gradually increased from 1 to 5 DAI (Fig. 3b). However, our previous report showed that the OSSUT1 transcript significantly increased after 1 to 2 DAI and then quickly decreased at 3 DAI (Chen et al. 2010). The expression level of OsSUT1 was the highest among OSSUT members in embryos at early imbibition stages (i.e., DAI 1), but OSSUT2 was the dominantly expressed OSSUT member 5 DAI (Fig. 3a). Thus, the functions and regulations of individual OsSUT genes are different in the embryos of germinating seeds. As shown by the data in Fig. 8, the expression levels of OSSUT2 in scutellum and vascular bundles were higher than in other embryo ground cells, and the OSSUT2 promoter activity was also significantly observed in aleurone layers. According to the OSSUT2 expression patterns in germinating seeds, it was suggested that OsSUT2 play a role to release sucrose from aleurone layers, transport sucrose into scutellum, and also load sucrose into the phloem in germinating seeds. On the other hand, OsSUT1 gene was expressed in the scutellar vascular bundle of germinating embryos but not in the scutellar epithelial cell layer. Therefore, it was suggested that OsSUT1 functioned to load sucrose into phloem for transport to developing shoot and roots but not play a role to transport sucrose from endosperm to embryos (Scofield et al. 2007).

The increased OSSUT2 expression in isolated embryos (without endosperms) during germination was not obvious in dark conditions; however, the significant up-regulation of OSSUT2 was observed in the embryos of germinating seeds (with endosperms) in dark conditions. Thus, it was suggested that the sugar transported from endosperm was the key factor for promoting OSSUT2 expression. Sugars not only act as nutrients and energy for supporting plant growth but also function as signals controlling plant development and the expression of various genes (reviewed by Gibson 2005; Rolland et al. 2006). The data in Fig. 6 showed that the transcript levels of rice OSSUT2 could be slightly enhanced by glucose and sucrose after 1 day of treatment and significantly enhanced after 5 days of treatment. Moreover, since the OSSUT2 expression was not affected by mannitol, the sugar-enhanced OSSUT2 expression was not caused by osmotic effect. Sugarenhanced expression has also been observed with OSSUT1 (Matsukura et al. 2000; Chen et al. 2010). However, the positive effect of sugar on OSSUT1 expression occurs after 5 days of treatment, and 1-day sugar treatment downregulates OSSUT1 expression (Chen et al. 2010). Thus, the mechanisms of sugar-mediated regulation are different for OsSUT1 and OSSUT2. Sucrose-induced signal transduction for gene regulation could involve sucrose as a direct signal that is sensed by a sensor located on the cell membrane or an intracellular sensor. In addition, sucrose metabolites, such as glucose, could be signals to trigger the downstream transduction pathway (reviewed by Halford et al. 1999). The nonmetabolizable sucrose analog palatinose had a similar effect on OSSUT2 expression in rice embryos as sucrose, suggesting that sucrose acts as a direct molecule for triggering the up-regulation of OSSUT2 expression. In addition, the sensor for sucrose signal transduction is expected to be located on the cell membrane because of the lack of palatinose transport into plant cells (Sinha et al. 2002; Rolland et al. 2006). Moreover, since there was no effect of mannitol on OSSUT2 expressions (Fig. 6), it was suggested that the up-regulation of OSSUT2 expressions by palatinose was not caused by osmotic effect. Moreover, the data showing that 3-OMG can also conduct the same positive effect on OSSUT2 expression in germinating embryos as glucose suggests that the glucose-induced OSSUT2 expression was mediated by a hexokinaseindependent pathway. In contrast, glucose-regulated OSSUT1 expression in germinating embryos was via a hexokinasedependent pathway (Chen et al. 2010).

Changes in the transcriptional activity of the OSSUT2 promoter in embryos during germination correlated with the mRNA levels. OSSUT2 promoter activity was also up- 
regulated by glucose in embryos. Thus, promoter regulation was a key step for controlling OSSUT2 expression. The predicted cis-acting elements on the OSSUT2 promoter were searched in the PLACE database (Higo et al. 1999), identifying a SUSIBA2 transcription factor-binding site (WBOXHVISO1; W-box) 477 bp upstream of the ATG translation start codon. SUSIBA2 is a sugar-inducible WRKY protein that can bind the sugar-responsive element on the barley isoamylase 1 promoter (Sun et al. 2003). Further study is needed regarding whether the interaction of SUSIBA2 and the W-box on the OSSUT2 promoter is a key factor to controlling sugar-responsive OSSUT2 expression.

In conclusion, we identified OsSUT2 as a plasma membrane transporter. OSSUT2 expression in germinated embryos correlates with the developmental stage, with regulation depending on the sugar transported from endosperms. The developmental regulation and signaling pathways of sugarresponsive OSSUT2 expression in germinating embryos are different from those of OsSUT1. Glucose-enhanced OSSUT2 expression was mediated by a hexokinase-independent pathway, and sucrose is sensed by a sensor located on the plasma membrane to up-regulate OSSUT2 expression. We also found that promoter activity is the major factor controlling the developmental stage- and sugar-dependent mRNA accumulation of OSSUT2 in the embryos of germinating seeds. Future studies of the activity of the deleted promoter element and finding the factors that interact with the cis-acting element would be helpful for elucidating the molecular mechanism of OSSUT2 expression in germinating rice embryos.

Acknowledgements We thank Dr. Chwan-Yang Hong from National Taiwan University for providing the pCHY10 and pCAMBIA1302 plasmids and Mr. Dah-Pyng Shung from Hualien District Agricultural Research and Extension Station in Taiwan for providing the rice seeds. This research was supported by grant NSC 99-2313-B-002-006-MY3 from the National Science Council of the Republic of China.

\section{References}

Aoki N, Hirose T, Scofield GN, Whitfeld PR, Furbank RT. The sucrose transporter gene family in rice. Plant Cell Physiol. 2003;44:223-32.

Aoki N, Scofield GN, Wang XD, Offler CE, Patrick JW, Furbank RT. Pathway of sugar transport in germinating wheat seeds. Plant Physiol. 2006;141:1255-63.

Barker L, Kühn C, Weise A, Schulz A, Gebhardt C, Hirner B, et al. SUT2, a putative sucrose sensor in sieve elements. Plant Cell. 2000;12:1153-64.

Bouteau F, Dellis O, Bousquet U, Rona JP. Evidence of multiple sugar uptake across the plasma membrane of lacticifer protoplasts from Hevea. Bioelectrochem Bioenerg. 1999;4:135-9.

Braun DM, Slewinski TL. Genetic control of carbon partitioning in grasses: roles of sucrose transporters and tie-dyed loci in phloem loading. Plant Physiol. 2009;149:71-81.
Bürkle L, Hibberd JM, Quick WP, Kühn C, Hirner B, Frommer WB. The $\mathrm{H}^{+}$-sucrose cotransporter NtSUT1 is essential for sugar export from tobacco leaves. Plant Physiol. 1998;118:5968.

Carpaneto A, Geiger D, Bamberg E, Sauer N, Fromm J, Hedrich R. Phloem-localized, proton-coupled sucrose carrier ZmSUT1 mediates sucrose efflux under the control of the sucrose gradient and the proton motive force. J Biol Chem. 2005;280:21437-43.

Chen JY, Liu SL, Siao W, Wang SJ. Hormone and sugar effects on rice sucrose transporter OSSUT1 expression in germinating embryos. Acta Physiol Plant. 2010;32:749-56.

Dixon M, Webb EC, eds. Enzymes. Longman, London. 1979; pp. 248-251.

Edelman J, Shibko SI, Keys AJ. The role of the scutellum of cereal seedlings in the synthesis and transport of sucrose. J Exp Bot. 1959;10:178-89.

Endler A, Meyer S, Schelbert S, Schneider T, Weschke W, Peters SW, et al. Identification of a vacuolar sucrose transporter in barley and Arabidopsis mesophyll cells by a tonoplast proteomic approach. Plant Physiol. 2006;141:196-207.

Gibson SI. Control of plant development and gene expression by sugar signaling. Curr Opin Plant Biol. 2005;8:93-102.

Halford NG, Purcell PC, Hardie DG. Is hexokinase really a sugar sensor in plants? Trends Plant Sci. 1999;4:117-20.

Hiei Y, Ohta S, Komari T, Kumashiro T. Efficient transformation of rice (Oryza sativa L.) mediated by Agrobacterium and sequence analysis of the boundaries of the T-DNA. Plant J. 1994;6:271-82.

Higo K, Ugawa $\mathrm{Y}$, Iwamoto $\mathrm{M}$, Korenaga T. Plant cis-acting regulatory DNA elements (PLACE) database:1999. Nuc Acids Res. 1999;27:297-300.

Hirose T, Imaizumi N, Scofield GN, Furbank RT, Ohsugi R. cDNA cloning and tissue specific expression of a gene for sucrose transporter from rice (Oryza sativa L.). Plant Cell Physiol. 1997;38:1389-96.

Jefferson RA. Assaying chimeric genes in plants: the GUS gene fusion system. Plant Mol Biol Rep. 1987;5:387-405.

Kahsay R, Liao L, Gao G. An improved hidden Markov model for transmembrane protein topology prediction and its applications to complete genomes. Bioinformatics. 2005;21:1853-8.

Kühn C, Grof CP. Sucrose transporters of higher plants. Curr Opin Plant Biol. 2010;13:288-98.

Kühn C, Quick WP, Schulz A, Reismeier JW, Sonnewald U, Frommer WB. Companion-cell-specific inhibition of the potato sucrose transporter SUT1. Plant Cell Environ. 1996;19:1115-23.

Lalonde S, Wipf D, Frommer WB. Transport mechanisms for organic forms of carbon and nitrogen between source and sink. Annu Rev Plant Biol. 2004;55:341-72.

Lemoine R. Sucrose transporters in plants: update on function and structure. Biochem Biophys Acta. 2000;1465:246-62.

Liu SL, Siao W, Wang SJ. Changing sink demand of developing shoot affects transitory starch biosynthesis in embryonic tissues of germinating rice seeds. Seed Sci Res. 2010;20:137-44.

Matsukura C, Saitoh T, Hirose T, Ohsugi R, Perata P, Yamaguchi J. Sugar uptake and transport in rice embryo. Expression of companion cell-specific sucrose transporter (OsSUT1) induced by sugar and light. Plant Physiol. 2000;124:85-93.

Mena M, Cejudo FJ, Isabel-Lamoneda I, Carbonero P. A role for the DOF transcription factor BPBF in the regulation of gibberellin-responsive genes in barley aleurone. Plant Physiol. 2002;130:111-9.

Murata T, Akazawa T, Fukuchi S. Enzymic mechanism of starch breakdown in germinating rice seeds. I. An analytical study. Plant Physiol. 1968;43:1899-905.

Nomura T, Nomura T, Akazawa T. Enzymic mechanism of starch breakdown in germinating rice seeds II. Scutellum as the site of sucrose synthesis. Plant Physiol. 1969;44:765-9. 
Rae AL, Perroux JM, Grof CPL. Sucrose partitioning between vascular bundles and storage parenchyma in the sugarcane stem: a potential role for the ShSUT1 sucrose transporter. Planta. 2005;220:817-25.

Reinders A, Schulze W, Kühn C, Barker L, Schulz A, Ward JM, et al. Protein-protein interactions between sucrose transporters of different affinities colocalized in the same enucleate sieve element. Plant Cell. 2002;14:1567-77.

Reinders A, Sivitz AB, Starker CG, Gantt JS, Ward JM. Functional analysis of LjSUT4, a vacuolar sucrose transporter from Lotus japonicus. Plant Mol Biol. 2008;68:289-99.

Rolland N, Ferro M, Seigneurin-Berny D, Garin J, Douce R, Joyard J. Proteomics of chloroplast envelope membranes. Photosynth Res. 2003;78:205-30.

Rolland F, Baena-Gonzalez E, Sheen J. Sugar sensing and signaling in plants: conserved and novel mechanisms. Annu Rev Plant Biol. 2006;57:675-709.

Scofield GN, Aoki N, Hirose T, Takano M, Jenkins CLD, Furbank RT. The role of the sucrose transporter, OsSUT1, in germination and early seedling growth and development of rice plants. J Exp Bot. 2007;58:483-95.
Sinha AK, Hofmann MG, Römer U, Köckenberger W, Elling L, Roitsch T. Metabolizable and non-metabolizable sugars activate different signal transduction pathways in tomato. Plant Physiol. 2002;128:1480-9.

Spackman VMT, Cobb AH. An enzyme-based method for the rapid determination of sucrose, glucose and fructose in sugar beet roots and the effects of impact damage and postharvest storage in clamps. J Sci Food Agri. 2002;82:80-6.

Stanley D, Rejzek M, Naested H, Smedley M, Otero S, Fahy B, et al. The role of alpha-glucosidase in germinating barley grains. Plant Physiol. 2011;155:932-43.

Sun C, Palmqvist S, Olsson H, Borén M, Ahlandsberg S, Jansson C. A novel WRKY transcription factor, SUSIBA2, participates in sugar signaling in barley by binding to the sugar-responsive elements of the iso1 promoter. Plant Cell. 2003;15:2076-92.

Toki S. Rapid and efficient Agrobacterium-mediated transformation in rice. Plant Mol Biol Rep. 1997;15:16-21.

Weise A, Barker L, Kühn C, Lalonde S, Buschmann H, Frommer WB, et al. A new subfamily of sucrose transporters, SUT4, with low affinity/high capacity localized in enucleate sieve elements of plants. Plant Cell. 2000;12:1345-55. 